\title{
DESIGN AND DEVELOPMENT AI-ENABLED EDGE COMPUTING FOR INTELLIGENT-IOT APPLICATIONS
}

\author{
Dr. D. Sivaganesan \\ Professor, Department of Computer Engineering \\ PSG Institute of Technology and Applied Research \\ Coimbatore, India \\ Email: sivaganesan@psgitech.ac.in
}

\begin{abstract}
The advancements in the technologies and the increase in the digital miniaturization day by day are causing devices to become smarter and smarter and the emergence of the internet of things and the cloud has made things even better with insightful suggestions for organization as well as the way the people work and lead their life. The limitations in the cloud paradigm in terms of processing complexity, the latency in the service provisioning and improper resource scheduling, remains as a reason leading to shifting of applications from cloud to edge. More over the emergence of the artificial intelligence in the edge computing has turned out to be center of attention as it improves the speed and the range of the IOT applications. The paper also puts forth the design of the AI-enabled Edge computing for developing a Smart Farming.
\end{abstract}

Keywords: Artificial Intelligence, Edge Computing, Internet of Things, Smart Farming, Accurate Resource Utilization

\section{INTRODUCTION}

The IOT devices growing at a rapid pace has increased the height of the data generation causing an enormous flow of information. These sensitive data gathered by the device associated with the IOT are been largely stored in the cloud paradigm but the delay experienced in the conveyance between the cloud data centers and the end-users has made the arrangement baseless. Even the organization associated with the cloud faces reliability issues while handling operational needs that are critical.

This has remained as the reason for the several enterprises or the organizations to shift from the cloud paradigm to edge that enables the information to be processed or executed locally at the user end allowing a faster computation and real time decision making.

ISSN: $2582-4104$ 
Journal of trends in Computer Science and Smart technology (TCSST) (2019)

Vol.01/ No. 02 Pages: 84-94

https://www.irojournals.com/tcsst/

DOI: https://doi.org/10.36548/jtcsst.2019.2.002

The edge computing is considered as the promising frame work that has side stepped the cloud by ensuring an automated decision making with very low latency and enabling a seamless gleaning of data form the devices associated with the IOT along with the real time decision making at the user end.

The emergence of the artificial intelligence has resulted with an accurate and an intelligent allocation of utilities and has become predominant in multitude of applications. The artificial intelligence incorporated into the edge has strengthened the edge computing with its compact structural design and ensures an improved decision making, allowing a huge amount of information to be stored locally eluding the necessity of storing the information in a remote place.

The utilization of edge computing eludes the entailment to convey the data to the cloud for executing/processing, thus eliminating the issue related to the latency and the time complexity. Moreover the advancements in the information technology and the communication have led to the save, compute and decide with the solution locally by constructing an on premise IOT solution. The Artificial Intelligence in the edge computing enables a continuous processing by avoiding the unexpected failures in the devices and identifying the requirement for the predictive maintenance. The AI more over ensure the secured repository for the sensitive information's by storing the information's locally within the premises and reduces the security threats. The fig .1 below provides the advantages of the edge enriched with the AI.

ISSN: $2582-4104$ 
Journal of trends in Computer Science and Smart technology (TCSST) (2019)

Vol.01/ No. 02 Pages: 84-94

https://www.irojournals.com/tcsst/

DOI: https://doi.org/10.36548/jtcsst.2019.2.002

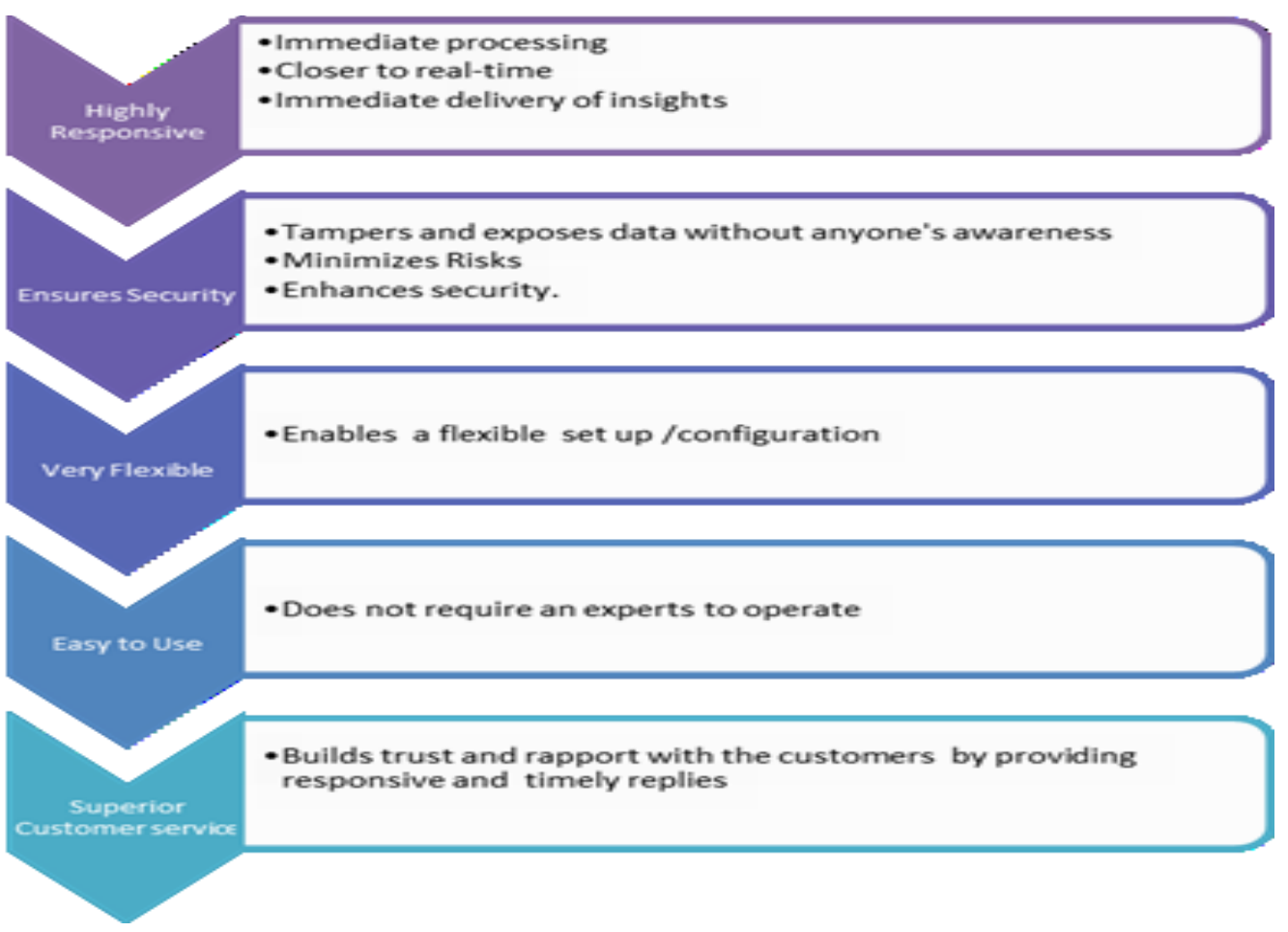

Fig .1 Advantages of Edge Enriched with AI

So the paper puts forth the AI enabled edge computing for examining the crop growth, soil moisture, pestilence attack, temperature, humidity, climatic changes, occurrence of disasters and the time to harvest, turning the farming smart without the intervention of the cloud computing.

The paper is organized with the related works in the section II, proposed AI enabled Edge computing for smart farming in section III, the results gained in Section IV and Conclusion in section V.

ISSN: $2582-4104$ 
Journal of trends in Computer Science and Smart technology (TCSST) (2019)

Vol.01/ No. 02 Pages: 84-94

https://www.irojournals.com/tcsst/

DOI: https://doi.org/10.36548/jtcsst.2019.2.002

\section{RELATED WORKS}

The edge computing behaving as a promising alternative for the cloud for the applications that requires an onpremises, storage and processing eluding the latency in execution /computing of the information's . The involvement of AI in the Edge has further enhanced the capability of the edge by providing a continuous monitoring and increasing the production rate. The Related work hold few recent papers that have proposed application with the cloud, fog, edge and the AI enabled edge. Venkatesh et al [1] in his paper explores the "The Evolution of Internet of Things: Bringing the power of Artificial Intelligence to IoT, its Opportunities and Challenges." and Duraipandian, M et al [2] in his paper provides the "Cloud Based Internet of Things for Smart Connected Objects."

Author Valanarasu et al [3] presents the "Smart and Secure Iot and Ai Integration Framework for Hospital Environment." The author S. Smys et al [4] and [5] details the "A novel report on architecture, protocols and applications in Internet of Things (IoT)" and elaborates the entailment of the "Inventive Network Structures for Next Generation Wireless Personal Systems." respectively. He also provides a detailed lecture on the "Wireless systems: New technologies, resource optimization and security" in his article [6].

Yamakami et al [7] puts forth the. "Experimental Implementation of an Edge-based AI Engine with Edge-Cloud Coordination." In his paper and Chen, et al [8] presents the "perspective of the AI in the mobile edge computing" Cui, et al [9] has offered the lecture on the approaches that "drive the AI empowered management" and FernándezCaramés et al [10]has put forward the "A Review on Blockchain, IoT, Fog and Edge Computing Enabled Smart Campuses and Universities for the Next Generation Teaching, Learning, and Context-Aware Applications for Higher Education"

Yang et al [11] has proposed "Edge Coordinated Query Configuration for Low-Latency and Accurate Video Analytics." Avoiding the intervention of the cloud and the latency in its computation. Rupanagudi et al [12] put forth the "involvement of the cloud computing in smart farming system for early detection of borer insects in tomatoes." Jagannathan et al [13] has put forth the ""Smart farming system using sensors for agricultural task automation." Pandian, et al [14] details the "ENHANCED EDGE MODEL FOR BIG DATA IN THE INTERNET OF THINGS BASED APPLICATIONS" in his paper.

ISSN: 2582-4104 
Journal of trends in Computer Science and Smart technology (TCSST) (2019)

Vol.01/ No. 02 Pages: 84-94

https://www.irojournals.com/tcsst/

DOI: https://doi.org/10.36548/jtcsst.2019.2.002

\section{PROPOSED WORK}

The proposed model of smart framing shifts from the traditionally used cloud computing paradigm to the edge computing to reduce the latency in the service provided, and the cost charged for the communication of the information. Any way the edge could not replace the cloud paradigm as it is has the capability of accommodating any number of service utilization and data storage for multitudes of users. But the edge could be a promising alternative in case of small level application that requires an on premises computation and solution with a limit period of time. So the smart farming in the proposed model prefers the edge computing to bring down the security threats, enhance the speed and minimize the cost.

The proposed frame work of the smart farming includes the artificial intelligence along with the edge computing to enhance the farming output and the efficiency, by enabling the farmer to completely monitor the crop health and soil status regularly. The block diagram in the Fig. 2 details the involvement of AI in the edge computing for the smart farming.

ISSN: 2582-4104 
Journal of trends in Computer Science and Smart technology (TCSST) (2019)

Vol.01/ No. 02 Pages: 84-94

https://www.irojournals.com/tcsst/

DOI: https://doi.org/10.36548/jtcsst.2019.2.002

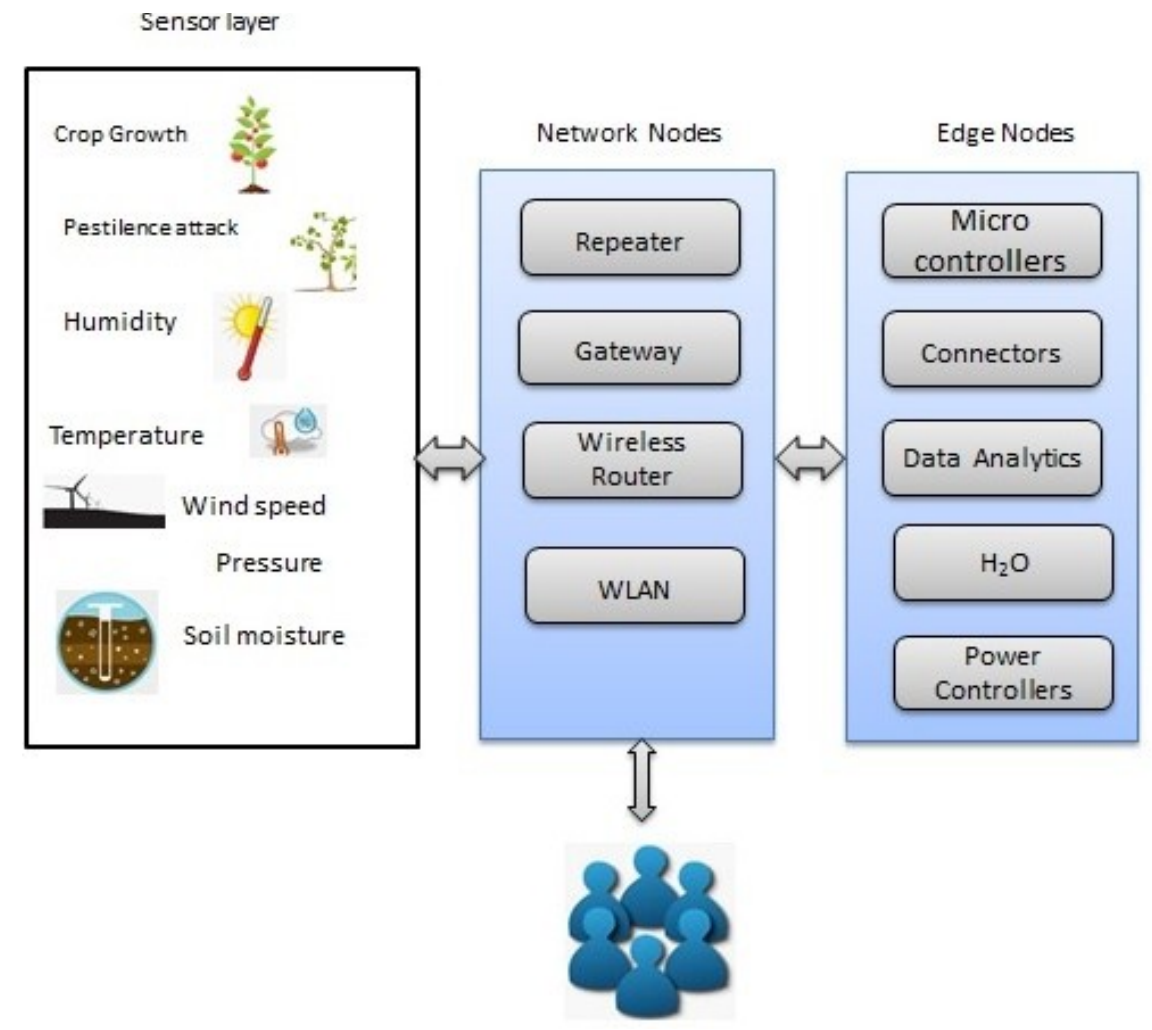

Fig.2 Frame Work of the Proposed

The artificial intelligence involved in the edge computing utilizes the deep learning module $\mathrm{H}_{2} \mathrm{O}$ to predict the consistency of the soil the growth of the crop and the proper time to sow as well as harvest. It also provides the weather forecast the natural disaster's to occur based on the dataset provided for training. It learns utilizing the multi-layer feed forward artificial neural network that is trained using the stochastic gradient that uses the back propagation algorithm. The ISTAT (national institute of statistics) dataset was used in training the neural network employing the back propagation algorithm and tested using the real time dataset gathered based on the crop growth, temperature, weather, humidity, forth coming disasters and the right time to sow and reap.

The drone enabled with the tetracam-ADC lite camera enables to monitor the growth in the crops and the pestilence attacks. The wireless environmental sensor engaged enables to monitor the temperature, humidity, soil moisture, the

ISSN: 2582-4104 
Journal of trends in Computer Science and Smart technology (TCSST) (2019)

Vol.01/ No. 02 Pages: 84-94

https://www.irojournals.com/tcsst/

DOI: https://doi.org/10.36548/jtcsst.2019.2.002

wind speed and the pressure etc., entire information of the farming area is completely gathered and transmitted to the edge layer where the information's are computed using the $\mathrm{H}_{2} \mathrm{O}$. Based on the training provided for the $\mathrm{H}_{2} \mathrm{O}$, it predicts the necessity for the immediate measure to be taken and alarms the farmers for the preparations to be done. The temperature, humidity, wind speed and the pressure sensor enable the deep learning module to predict the right time to sow as well as reap. The soil moisture sensor enables to have the right time water the crops and the drones examining the crop growth and the pestilence attack enables to know the right time to add the fertilizers to the crop to avoid the damages in the crop.

All the information gathered from the sensor is conveyed using the Wi-Fi max and processed in the edge layer and conveyed back to the portable device of the farmer using the Wi-Fi. This reduces the time consumption in the conveyance of the information as well as the delay in the execution of the task.

\section{RESULTS}

The proposed model is tested based on the real time data set observed to evince the accuracy of the prediction and evaluated using the MATLAB to note down the maximum time consumed in transmission of the information from the edge to the user. The fig. 3 shows the percentage of the accuracy in prediction based on the details observed for a week.

ISSN: 2582-4104 
Journal of trends in Computer Science and Smart technology (TCSST) (2019)

Vol.01/ No. 02 Pages: 84-94

https://www.irojournals.com/tcsst/

DOI: https://doi.org/10.36548/jtcsst.2019.2.002

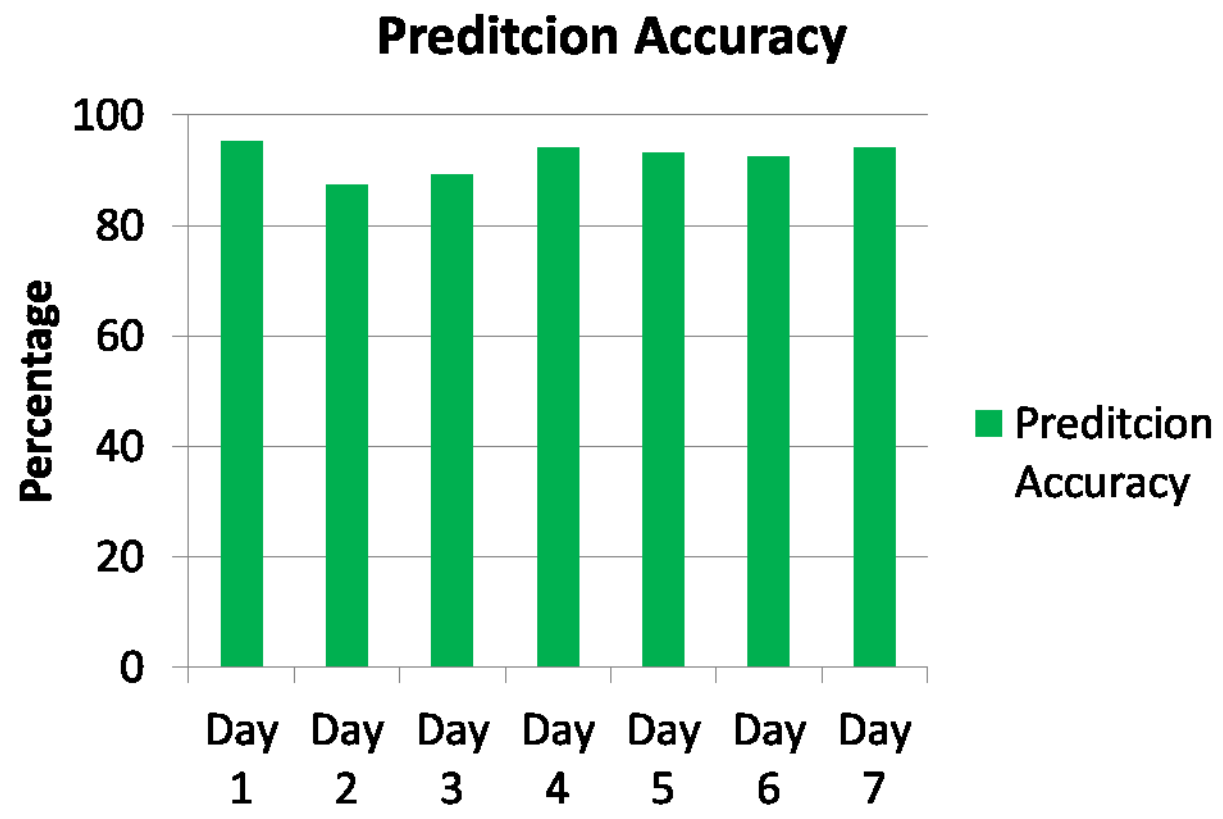

Fig.3 Prediction Accuracy

The fig .4 below provides the latency observed in information conveyance from the edge to the user for a week.

ISSN: $2582-4104$ 
Journal of trends in Computer Science and Smart technology (TCSST) (2019)

Vol.01/ No. 02 Pages: 84-94

https://www.irojournals.com/tcsst/

DOI: https://doi.org/10.36548/jtcsst.2019.2.002

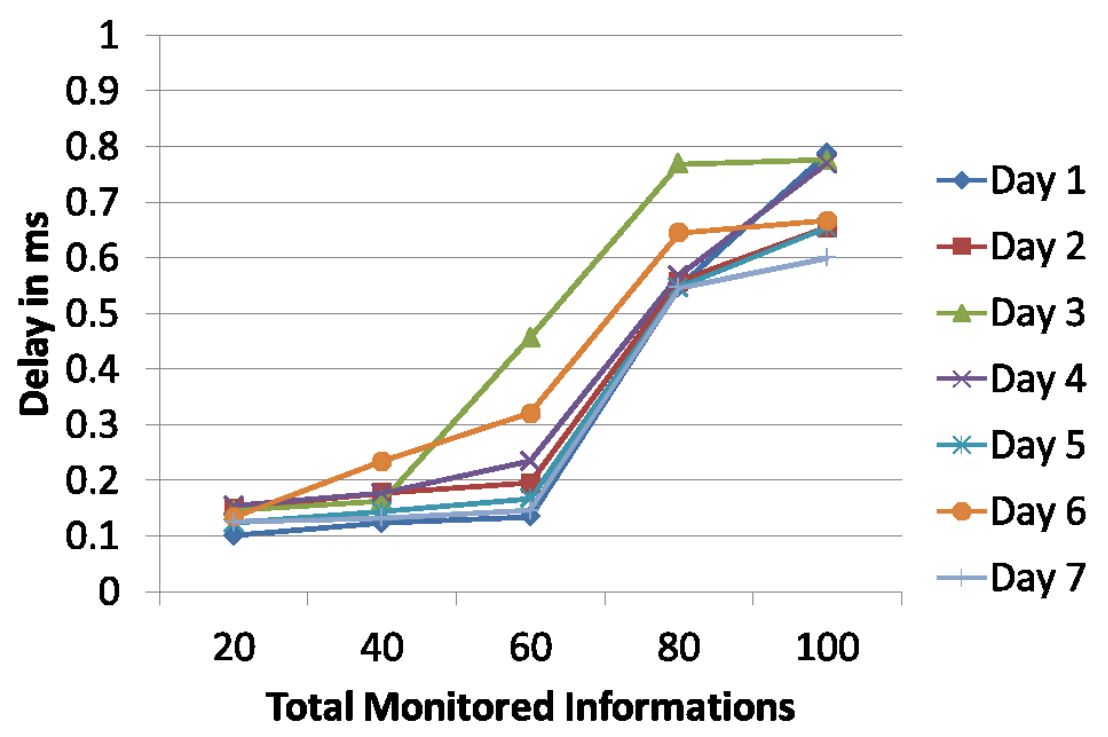

Fig.4 Time consumed

\section{CONCLUSION}

The Edge computing enabled with the artificial intelligence could be accessed from anywhere at any time without the intervention of the cloud, it would be highly suitable for the small size application that requires an on premises solution with a secure information storage and reduced latency in processing. So the AI enabled edge computing could not be a replacement for the cloud computing that can handle a huge set of data and interconnect the entire world. The proposed model with AI enabled edge computing for the smart computing is an on premises solution for the agriculture field by providing the accurate information's of the soil moisture, temperature, humidity etc. related to the particular field. The AI enabled Edge ensure the effective way to decrease the unexpected failure of the devices allowing a continuous monitoring and increase in the productivity by providing the accurate information's at very low latency. The results obtained shows that the smart farming with the AI enabled edge has a limited power consumption and conveyance time on comparing with the traditional methods. In future the paper is to continue with survey on the AI enabled automation methods involved in the plough-ing, sowing, harvesting and the supply chain management of the crops.

ISSN: $2582-4104$ 
Journal of trends in Computer Science and Smart technology (TCSST) (2019)

Vol.01/ No. 02 Pages: 84-94

https://www.irojournals.com/tcsst/

DOI: https://doi.org/10.36548/jtcsst.2019.2.002

\section{References}

[1] Venkatesh Naganathan, Prof, and Rajesh Rao. "The Evolution of Internet of Things: Bringing the power of Artificial Intelligence to IoT, its Opportunities and Challenges."

[2] Duraipandian, M., and Mr R. Vinothkanna. "CLOUD BASED INTERNET OF THINGS FOR SMART CONNECTED OBJECTS." Journal of ISMAC 1, no. 02 (2019): 111-119.

[3] Valanarasu, Mr R. "SMART AND SECURE IOT AND AI INTEGRATION FRAMEWORK FOR HOSPITAL ENVIRONMENT." Journal of ISMAC 1, no. 03 (2019): 172-179.

[4] Kumar, R. Praveen, and S. Smys. "A novel report on architecture, protocols and applications in Internet of Things (IoT)." In 2018 2nd International Conference on Inventive Systems and Control (ICISC), pp. 11561161. IEEE, 2018.

[5] Smys, S., and Robert Bestak. "Introduction to the Special Issue on Inventive Network Structures for Next Generation Wireless Personal Systems." Wireless Personal Communications 90, no. 2 (2016): 421-422.

[6] Shadaram, Mehdi, S. Smys, and Sherali Zeadally. "Introduction to the special issue on "Wireless systems: New technologies, resource optimization and security"." Computers and Electrical Engineering 2, no. 40 (2014): 289-290

[7] Yamakami, Toshihiko. "An Experimental Implementation of an Edge-based AI Engine with Edge-Cloud Coordination." In 2018 18th International Symposium on Communications and Information Technologies (ISCIT), pp. 442-446. IEEE, 2018.

[8] Chen, Zhuang, Qian He, Lei Liu, Dapeng Lan, Hwei-Ming Chung, and Zhifei Mao. "An Artificial Intelligence Perspective on Mobile Edge Computing." In 2019 IEEE International Conference on Smart Internet of Things (SmartIoT), pp. 100-106. IEEE, 2019.

[9] Cui, Shuguang, Liuqing Yang, and Xiang Cheng. "Guest Editorial: Special Issue on AI Powered Network Management: Data-Driven Approaches Under Resource Constraints." IEEE Internet of Things Journal 5, no. 6 (2018): 4233-4236.

[10] Fernández-Caramés, Tiago M., and Paula Fraga-Lamas. "Towards Next Generation Teaching, Learning, and Context-Aware Applications for Higher Education: A Review on Blockchain, IoT, Fog and Edge Computing Enabled Smart Campuses and Universities." Applied Sciences 9, no. 21 (2019): 4479.

[11] Yang, Peng, Feng Lyu, Wen Wu, Ning Zhang, Li Yu, and Xuemin Shen. "Edge Coordinated Query Configuration for Low-Latency and Accurate Video Analytics." IEEE Transactions on Industrial Informatics (2019).

ISSN: $2582-4104$ 
Journal of trends in Computer Science and Smart technology (TCSST) (2019)

Vol.01/ No. 02 Pages: 84-94

https://www.irojournals.com/tcsst/

DOI: https://doi.org/10.36548/jtcsst.2019.2.002

[12] Rupanagudi, Sudhir Rao, B. S. Ranjani, Prathik Nagaraj, Varsha G. Bhat, and G. Thippeswamy. "A novel cloud computing based smart farming system for early detection of borer insects in tomatoes." In 2015 International Conference on Communication, Information \& Computing Technology (ICCICT), pp. 1-6. IEEE, 2015.

[13] Jagannathan, S., and R. Priyatharshini. "Smart farming system using sensors for agricultural task automation." In 2015 IEEE Technological Innovation in ICT for Agriculture and Rural Development (TIAR), pp. 49-53. IEEE, 2015.

[14] Pandian, A. Pasumpon. "ENHANCED EDGE MODEL FOR BIG DATA IN THE INTERNET OF THINGS BASED APPLICATIONS." Journal of trends in Computer Science and Smart technology (TCSST) 1, no. 01 (2019): 63-73

\footnotetext{
ISSN: 2582-4104
} 